\title{
IFR OPERATIONS AT NON-TOWERED, NON-RADAR AIRPORTS: CAN WE DO BETTER THAN ONE-AT-A-TIME?
}

\author{
K. Jones ${ }^{*}$, D. Williams ${ }^{\dagger}$, M. Consiglio ${ }^{\ddagger}$, and C. Adams ${ }^{\S}$ \\ NASA Langley Research Center, Hampton, VA \\ T. Abbott ${ }^{\dagger}$ \\ Booz Allen Hamilton, McLean, VA
}

\begin{abstract}
$\underline{\text { Abstract }}$
This paper describes a new concept for operations in non-radar "terminal" airspace around small, nontowered airports. Currently, air traffic operations in instrument meteorological conditions (IMC) at airfields without control towers and radar service are severely constrained by what is known as the "one-in/one-out" paradigm. Under these conditions only one operation (either arrival or departure) is allowed to occur at a time. Since these operations can take over 15 minutes to complete, capacity at these airports is severely restricted in IMC. The proposed concept is an attempt to break this current paradigm by applying emerging airborne and ground-based technologies to enable simultaneous operations by multiple aircraft in nonradar "terminal" airspace around small non-towered airports in IMC. The general philosophy underlying this concept of operations is the establishment of a newly defined area surrounding these airports called a Self-Controlled Area (SCA). Aircraft operating within the SCA are required to have a specified minimum level of equipage. Within the SCA, pilots are responsible for separating themselves from other similarly equipped aircraft through the use of new onboard systems and procedures. This concept also takes advantage of newly developed automation at the airport, which provides appropriate sequencing information to the pilots for safe and improved operations. Such operations would enhance the opportunity for point-to-point air taxi or charter operations into smaller airfields that are closer to a traveler's origin and destination. A description of this concept of operations and a simulation environment used for evaluation is provided in this paper.
\end{abstract}

\section{$\underline{\text { Introduction }}$}

Over the years, Americans have come to depend on our national air transportation system for the efficient and

\footnotetext{
* Senior Research Engineer, Senior Member AIAA

${ }^{\dagger}$ Senior Research Engineer

* Senior Research Engineer, Member AIAA

$\S$ Research Psychologist
}

rapid movement of people, goods, and services. This system has evolved from the airmail routes of the early 1900 's to a means of speeding packages ordered over the internet to appropriate distribution points. It has evolved from travel only for the affluent, to today's necessary means of travel for business and leisure. In the United States alone in 2000, there were more than 670 million enplanements and more than 670 billion revenue passenger miles. ${ }^{1}$ Commercial air transport service has become so important to our lives that any major disturbance in its service is met by public outcry and is reported on the nightly news.

While the current system of hub and spoke operations has served us well for many years, it is beginning to reach a capacity plateau. Due to the increasing demand on the system and with only modest potential gains in capacity, the system will reach gridlock within the next 10-15 years. Gridlock of this magnitude will have serious implications on the health of our economy. ${ }^{1,2,3}$ Additionally, the loss of non-stop flights as a result of the airlines more economical hub and spoke system, means that people are traveling significantly farther or longer to get to their destination. Nearly $70 \%$ of domestic air travelers are forced to fly through fewer than 35 of the Nation's more than 18,000 landing facilities. These intermediate stops and their accompanying layovers dramatically increase a traveler's overall door-to-door trip time. The travel time from Norfolk to Orlando is inconveniently extended by a lengthy layover at a hub airport where additional passengers are collected from flights originating in other cities. The success of air carriers that specifically target more point-to-point travel is evidence that people are seeking greater mobility through more convenient and less costly alternatives for air service.

A number of organizations have realized the deficiencies in today's system and have taken steps to address them. The FAA's Operational Evolution Plan $(\mathrm{OEP})^{4}$ focuses on improving capacity and efficiency in the National Airspace System over the next ten years. NASA, in addition to consulting with the FAA on their OEP, is also looking at improving today's system through research being conducted under the Advanced Air Transportation Technologies Project. NASA is 
pursuing another, complementary approach via the Small Aircraft Transportation System (SATS) Project. SATS is exploring the feasibility of increasing personal mobility and system capacity by expanding access to more than 5,000 underutilized smaller airports that exist in the United States. While only $22 \%$ of the United States population lives within 20 miles of a major/hub airport and $41 \%$ live within 20 miles of any commercial airport, $93 \%$ of the population lives within 20 miles of a general aviation or regional airport. ${ }^{1}$ Many of these airports have no control towers and lie outside air traffic control radar coverage. These airports are not suitable, without significant investment, for use by today's airlines. However, they do provide a unique potential for convenient access and service to small cities and communities across the country. New, small, efficient aircraft being developed by companies such as Eclipse, Safire, Adam Aircraft and others, could provide point-to-point unscheduled and scheduled "airtaxi" transportation. The new access and mobility that this would create is important to community vitality and economic opportunity that increasingly depend on access to rapid point-to-point transportation.

The goal of the five-year SATS Project is to develop and evaluate technologies and capabilities that enable accessibility for small aircraft in near all weather conditions to virtually any small airport. The technologies targeted for development are aimed at smaller aircraft used for personal and business transportation, focusing on several enabling operational capabilities that are not possible in the current National Airspace System (NAS) environment. The work described in this paper focuses on enabling higher volume operations at these underutilized airports through simultaneous operations by multiple aircraft in instrument meteorological conditions (IMC). The concept goal is to demonstrate a minimum of two concurrent operations in the terminal area of a nontowered, non-radar airport.

The minimum goal of two aircraft operating simultaneously may not appear significant and certainly pales in comparison to the efficiency of today's high capacity, high density airports with sophisticated air traffic management and surveillance capabilities. In contrast, many of the airports that the SATS project is targeting are in airspace that lack radar coverage and often have no control tower or surveillance. In this environment, ATC is forced to use what is known as procedural separation (non-radar) that restricts operations to only one aircraft operating in the area at a time. Therefore, the importance of the SATS goal is to break the one-in/one-out paradigm and expand capacity by allowing multiple, simultaneous operations while achieving a level of safety equal to today's system.

\section{Today's Non-Radar Environment}

The question posed by the title - can we do better than one-at-a-time? - focuses on Instrument Flight Rules (IFR) aircraft separation requirements in non-radar, terminal airspace. In today's IFR environment, aircraft separation services provided by ATC can be classified as either radar (target-to-target) or procedural (target-toairspace). Often, procedural (non-radar) separation in the terminal airspace can only be provided to IFR aircraft by ensuring that there are no other IFR flights operating within the airspace around the airport, i.e. the airspace is considered to be "sterile". All additional requests for operations at the airport are delayed until the ongoing IFR arrival or departure is complete, hence the phrase "one-in/one-out," and the one-at-a-time operation.

In this one-in/one-out situation, departing flights are restricted to an ATC-specified departure time window, during which the airspace for this operation is sterilized of other IFR traffic. If this departure window is missed, the clearance becomes void and a new clearance must be requested. Once radar separation or some other means of procedural separation is possible on the departing aircraft, the airport airspace becomes available for use by other aircraft. For arriving aircraft, the same principles apply, though the "one-in/one-out" window normally begins with the loss of radar contact, which typically occurs during the descent for the approach, and ends with the pilot closing or canceling his flight plan upon landing.

Newly arriving aircraft requesting an approach while another IFR approach or departure operation is underway, will typically be required to hold at some location or altitude that does not interfere with the ongoing operation. As the airspace becomes available, holding aircraft are then normally given approach clearances on a first-come, first serve basis.

While procedural separation is safe and predictable, it severely restricts the number of operations at these airports. That restriction is usually not a significant problem because these airfields often have relatively light traffic loads during IMC. To be able to use of these airfields as an attractive alternative for increasing access and mobility, we must be able to do better than the procedural, one-at-a-time operation.

\section{Enabling Technology}

There have been a number of recently developed technologies that could be exploited for improving today's system. The primary deficit at these airports is the lack of surveillance data on aircraft operating in the terminal area. Through the Capstone project in Bethel Alaska, the FAA has demonstrated that technologies 
such as Automatic Dependent Surveillance-Broadcast (ADS-B) can be used to provide surveillance data on equipped airplanes. When combined with appropriate cockpit tools, such as Cockpit Display of Traffic Information (CDTI), pilots of these aircraft can obtain better situation awareness of the location of other similarly equipped airplanes. The FAA is also using a ground-based receiver located at Bethel to receive and then relay ADS-B data to Anchorage Center. ATC then uses that information and available radar information to provide separation services. $^{5}$

ADS-B technology is also being exploited in the development of airborne self-separation tools. One concept uses a combination of airborne-based Conflict Detection and Resolution (CD\&R) algorithms and a CDTI. This combination allows pilots to separate themselves from other similarly equipped traffic. These CD\&R algorithms can identify when a conflict (defined as a predicted loss of separation) is going to occur, provide the pilot with an alert (generally visual and aural), and provide a proposed resolution to the conflict. ${ }^{6,7}$ Another application of ADS-B technology has been the development of airborne-based, in-trail self-spacing tools for use in the terminal area, which are being explored by both the FAA's Safe Flight 21 Program and NASA's AATT Project. ${ }^{8,9}$ While much of the current research, development, and evaluations have been focused on air transport aircraft, these technologies and concepts also provide a starting point for SATS operational concept development.

\section{$\underline{\text { Previous Research }}$}

Automated solutions for IFR operations have been proposed that are based on ground-based separation and sequencing services to approaching and departing traffic at non-towered, non-radar airports. Most of these attempts depend on 4-D (lateral, vertical and time determinate) flight path prediction. ${ }^{10,11}$ Certification of such systems along with the cost and complexity of both the ground technology and the airborne equipage required to support it have proved to be a major challenge.

A much less ambitious automation system was described recently by Conway and Consiglio ${ }^{12}$ and is based on existing non-radar "one-in/one-out" IFR procedures. This method, referred as the Automated Airport Control Volume (AACV) model, has the advantage of simplicity, relatively low cost, and employs existing procedural separation rules. A block of airspace, the Airport Control Volume (ACV), is established around the airport where a ground based automated sequencing facility would manage access, allowing one aircraft in the ACV airspace at a time. The resulting operational concept is a "hybrid system of rule-based maneuvering, airborne self-separation and ground control" with low cost and complexity, as well as easy integration into the current ATC structure. The AACV concept is aimed at low volume airports where optimizing capacity is not a concern; therefore it places few constraints on participating aircraft. As a result, this approach offers a conceptual base from which to build more sophisticated systems, allowing the introduction of self-separation technologies using a combination of procedures and specialized tools while facilitating the transition towards truly distributed air traffic control. Experience with these systems in the low volume environment of the AACV could be used to validate the technology before attempts are made to adapt these systems into higher-volume terminal areas.

Still another approach to improving operations at nontowered, non-radar airports was pursued by Cain. ${ }^{13}$ Cain looked at using a combination of enabling technologies, a simplified GPS T approach designed in accordance with today's Terminal Arrival Area $(\text { TAA })^{14}$ design criteria, and automating the FAA's timed approach concept. ${ }^{15}$

Both the work by Conway and Consiglio and the work by Cain were helpful starting points for the current concept on improving operations at these airports and attempting to answer the question of "can we do better than one operation at a time?".

\section{Concept of Operations}

\section{Concept Overview}

The general philosophy underlying this concept of operations is the establishment of a newly defined area of flight operations called a Self-Controlled Area (SCA). During periods of IMC, a block of airspace will be established around designated non-towered, nonradar airports. Aircraft flying enroute to one of these SATS designated airports would be on a standard IFR flight plan with ATC providing separation services. Once they have entered the SCA, pilots would take responsibility for separation assurance between their aircraft and other similarly equipped aircraft. Using onboard equipment and procedures, they would then approach and land at the airport. Departures would be handled in a similar fashion. Details of the transition procedures for entering and departing the SCA will be discussed in a later section.

While pilots are required to take responsibility for selfseparation within the SCA, they are not required or allowed to take responsibility for sequencing their departures or arrivals within the SCA. Our concept would take advantage of a proposed ground-based 
automation system, called an Airport Management Module (AMM) that provides sequencing information to pilots for safe and improved operations. The AMM is typically located at the airport and will make these assignments based on calculations involving aircraft performance and position information, winds in the terminal area, missed approach requirements, and a set of predetermined operating rules for the SCA.

Because the SATS project is focused on a realistic, operationally deployed system in the 2010 timeframe, this concept must emphasize integration with current and planned near-term NAS operations and systems. As a result, the design approach must focus on simplicity from both a procedural and systems requirements standpoint. It is further assumed that any additional ATC workload must be minimized and that enroute procedures be as similar to today's system as possible. This concept is based on a distributed decision-making environment that will provide pilots the necessary procedures, airborne systems, traffic awareness, and aircraft sequence information to enable safe operations within the SCA while minimizing the requirements of the ground support tools. While this is a distributed decision-making environment, much of the decisionmaking is left with the pilot (as it is with VFR operations into these non-towered airports today). Finally, our overall philosophy is to try to keep things as simple and safe as possible.

\section{Self-Controlled Area Characteristics}

For the purposes of this paper, a generic SCA that will support a SATS environment is described. The geometry and configuration of a specific SCA would have to be uniquely defined for each designated airport. The airspace would meet current FAA airspace design criteria and comply with required standards for terrain avoidance, obstacle clearance, local traffic densities, and noise abatement procedures. The SCA is similar in concept to a class E surface area and is similar both in size and shape to concepts proposed by Conway and Consiglio $^{12}$ and by Cain ${ }^{13}$. Figure 1 is a drawing of the plan view of a generic SCA. All the waypoints shown are waypoints that exist today for a generic RNAV GPS-T approach. In our concept we are proposing to use the center Initial Approach Fix (IAF) on the $\mathrm{T}$ (BOBBY) for arrivals. The outrigger IAF's on the departure side of the T (ELLEN and GINNY) will be used both for Missed Approach Holding Fixes (MAHF) and as Departure Fixes (DF). Due to the nominal location of these waypoints, and the requirement to protect the airspace around the holds at these waypoints, the SCA would have a radius of approximately $15 \mathrm{~nm}$. This is a generic size and shape and no attempts to optimize the SCA have been investigated for this paper.

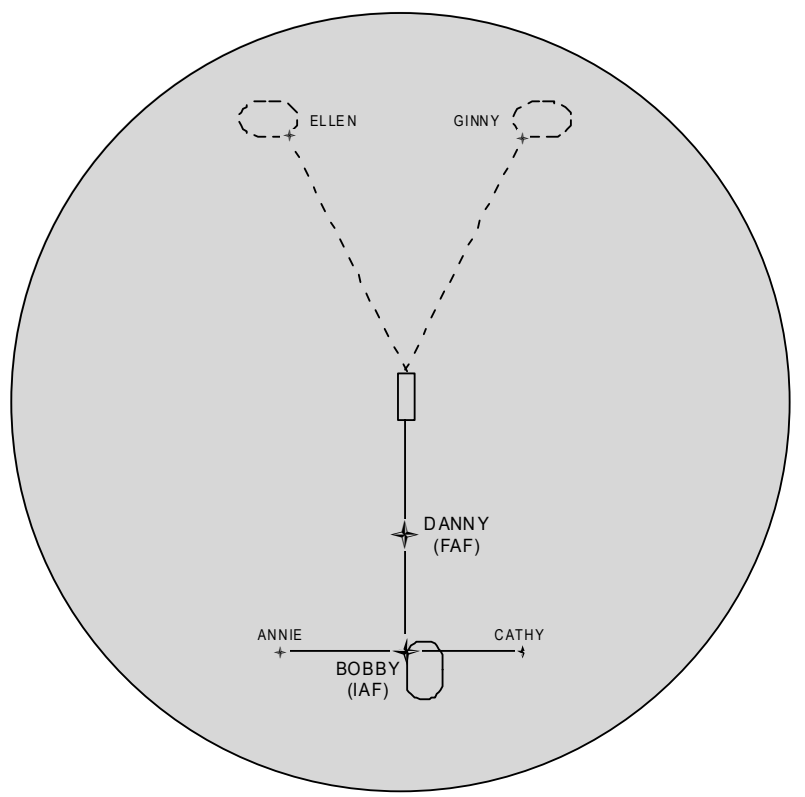

Figure 1. Plan view of a Self-Controlled Area

The concept of operations employed within the SCA is roughly based on the FAA's timed approach procedures, which can be utilized today at airfields with a control tower. In the current FAA procedure, the holding patterns and procedures are often compared to a stack of records. Arriving aircraft enter at the top of the stack, and then drop down in 1,000-foot increments when clear, until they reach the bottom of the stack, at which time they are cleared down the final approach for landing. Each pilot in the approach sequence is given advance notice of the time to leave the holding point and proceed onto the final approach. When the designated time to leave the holding point has been received, the pilot should adjust the flight path to leave the fix as closely as possible to that time. The purpose of the holding patterns is to delay aircraft while waiting clearance for the final approach and landing.

The SATS concept does not depend on a control tower or designated times but rather allows the pilot, using onboard equipment, to descend and then follow the preceding aircraft as designated by the AMM. The onboard equipment is used to verify that the altitude and location the pilot is descending to is free of other traffic. Pilots continue down the stack until they arrive at the initial approach altitude (2000 feet) at the IAF (BOBBY). Once additional requirements are met, the pilot leaves the hold at the IAF and continues down the approach. As a result of this concept, the height of the SCA is set nominally at 3000 feet above the airport with holds placed in the SCA at the center IAF (BOBBY) at 2000 and 3000 feet. The profile view in figure 2 shows these holds and helps visualize the stack above the IAF. Note also that the shape of the SCA is 
similar to a class $\mathrm{C}$ airspace design. Under certain conditions, VFR flight may be allowed at altitudes up to 700 feet above ground level. The SCA is sized so as to not penalize these operations except in the immediate vicinity of the airport. Again, the altitudes proposed for this airspace are nominal. Other altitudes and configurations can be established based on proper analysis and design constraints.

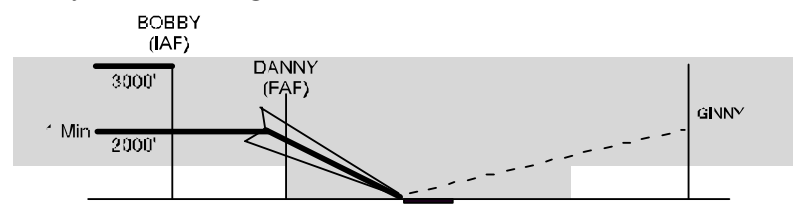

Figure 2. Profile view of a Self-Controlled Area

This concept further assumes that pilots have the ability to contact ATC prior to entering and leaving the SCA. While it is not required, it is assumed that airspace outside of the SCA is under radar coverage by ATC. Although procedural separation can be used for aircraft entering and arriving the SCA, if radar coverage is available adjacent to the ceiling of the SCA, transitions could be handled more efficiently. Outside of the SCA the airspace is "owned" by ATC and they are responsible for providing separation services.

\section{Airport Management Module Characteristics}

As noted above, the AMM consists of a ground-based automation system, typically located at the airport. It is responsible for determining aircraft sequencing for departures and arrivals. The AMM is not an automation of a tower controller but is more of a simple counter that issues sequence information based on a set of predetermined rules. The AMM relies on aircraft position information provided through a ground-based ADS-B transceiver to manage the operations within the SCA. Pilots will be expected to contact the AMM via data link and request landing or takeoff sequencing information. The AMM will then provide either a notification of which airplane the pilot is to follow (if there is anyone in the sequence ahead of the pilot) or inform the pilot that he cannot initiate the operation (entering or departing the SCA) along with a notification of the delay to expect before the operation can begin.

In addition to the other calculations the AMM must perform, the AMM must assure that there is available missed approach airspace for each aircraft that is arriving in the SCA. Since we must assume that every approach may result in a missed approach and since there is not an active controller involved in SCA operations (who could respond in real time with unique missed approach instructions), each pilot entering the SCA will be assigned specific missed approach information by the AMM as they enter the airspace.
This technique keeps the ground-based automation relatively simple and less critical to the operational concept. However, it does mean that the total number of operations is constrained by the number of unique missed approach locations that can exist within the SCA. For the SCA shown in figures 1 and 2, since there are four missed approach locations (2 each at the MAHF's), there are a total of 4 operations allowed at one time in this version of an SCA. Again, it is expected that this design would be modified for specific airport and airspace configurations. Also note, however, that significantly more missed approach locations may not significantly increase the number of allowable operations.

As mentioned previously, a number of predetermined rules for the SCA must be built into the AMM software. The following rules apply to this SCA concept.

1) The maximum number of concurrent operations in the SCA must be no more than four.

2) Upon entering the SCA at the IAF, aircraft are to go to the lowest available altitude and continue descending when altitudes below them become available.

3) Alternating missed approach holding fixes are given to sequential aircraft (ie., first aircraft is given ELLEN, second aircraft is given GINNY, third aircraft is given ELLEN, etc.)

4) When proceeding to a holding fix on a missed approach, aircraft are to go to the lowest available altitude (ie., first aircraft heading to ELLEN goes to 2000 feet, the next aircraft going to ELLEN goes to 3000 feet).

5) Aircraft operating in the SCA must be able to climb at 300 feet per mile (required for maintaining separation when climbing to the highest altitude of a MAHF or DF when the lower altitude is occupied).

6) Departing aircraft are held on the ground if the departure fix they are requesting already has two missed approaches assigned to that fix

7) Departing aircraft always climb to the highest available altitude at the departure fix. Additionally, aircraft must meet an altitude crossing restriction on the climb out to the DF's highest altitude to avoid conflict with any aircraft holding at lower altitudes (eg., must be at or above 3000 feet 10 miles from the airport).

Airports would only need to make relatively minimal infrastructure investments to increase operations during periods of IMC. Airports would be expected to have weather reporting capability (e.g., AWOS) and would 
have to install an AMM, a ground based ADS-B transceiver, and have a data link capability.

For this operational concept to be viable, a link between the AMM and ATC would be desirable. If controllers had access to the same information that pilots were being given, this could facilitate airspace management. For example, if controllers knew that the SCA was not currently accepting aircraft (because the SCA was full), and that there would be a 20-minute delay at the airport; they could begin planning for that delay in advance. Similarly, if they knew exactly when the AMM was going to release an airplane for departure, they would have greater insight as to when that airplane was going to show up on their display.

Aircraft requirements

For aircraft to be permitted to perform SATS operations within the SCA, they must be suitably equipped. As a minimum, aircraft must have an approach-certified IFR GPS receiver, an ADS-B transceiver, a communications data link, and a cockpit display of traffic information (CDTI). The specific type of CDTI is not enumerated here but it is envisioned that the CDTI will be capable of graphically depicting both a plan and profile view of ownship position, planned routing, and information on other traffic.

Since pilots operating within the SCA are responsible for performing the task of self-separation, they must be given appropriate cockpit tools to enable them to perform this task without significantly increasing their workload. Therefore, aircraft must not only be equipped with a CDTI but they must also have onboard conflict detection and alerting capability. Conflict detection tools compare the current flight path of one's own aircraft with those of any proximate aircraft to detect any potential or predicted loss of separation. Conflict alerting may be provided using visual and/or aural queues. The airplane may also be equipped with a selfspacing tool, which could enable the pilot to perform even more efficient operations.

Normal operations

To better envision this operational concept, the following is a description of a "typical" SATS operation.

Arrivals - No unique SATS provision is anticipated for filing and following flight plans so the pilot is simply required to file a traditional IFR flight plan to the SATS destination airport. The aircraft special equipment designator on the flight plan identifies the aircraft as SATS capable and would be available for use by ATC. The final fix in the route of flight section of the flight plan is the SATS transition fix, which is an
IAF for a SATS instrument approach at the destination SATS designated airport (eg., BOBBY). Prior to reaching the transition fix, the pilot requests a landing sequence from the AMM. If one is available, the AMM determines a preliminary sequence for the arriving aircraft relative to other aircraft already in the SCA. If the SCA is "full," the AMM issues expected delay times to the SATS aircraft. The delay times can be used by the pilot for working with ATC and in considering alternate airport options. The time given includes any expected delay for entering the SCA based on the time it is anticipated for a space in the SCA to open up and any expected delay for the approach.

ATC will clear the SATS aircraft to the SATS transition fix according to the flight plan. If there is no opening in the SCA, the SATS aircraft will need to hold at an altitude above the SCA (figure 3). Once the airspace is available, the AMM issues a clear-to-follow notification (CTFN) to the SATS aircraft. Rather than providing a constantly changing sequence number, the AMM indicates relative sequence by providing the pilot with the identification of an aircraft to follow. If there is no one to follow, the pilot receives a "clear" message. In addition to the CTFN message, the AMM also provides a missed approach holding fix assignment that is procedurally deconflicted from other SCA aircraft. The SATS pilot is required to acknowledge receipt of these messages.

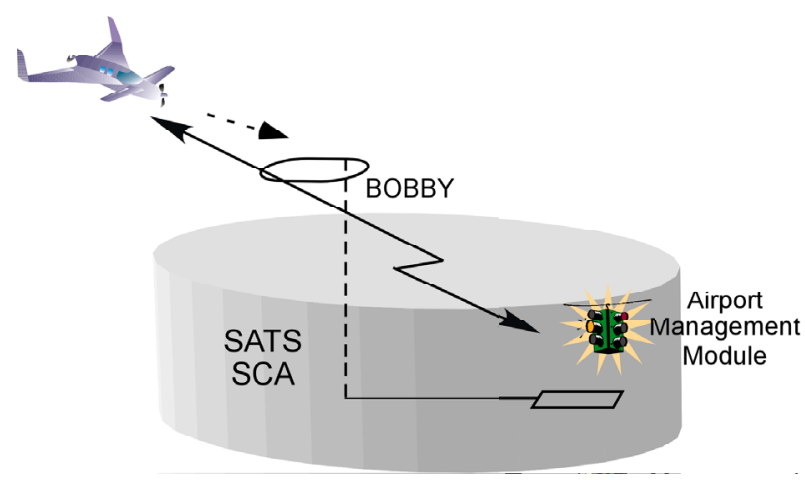

Figure 3. ATC clearance to the transition fix

Once these messages have been received and acknowledged, the pilot confirms, via onboard displays, that he is sufficiently clear from other traffic already within the SCA. The pilot then requests a descent into the SCA from ATC. ATC approves the descent when able and indicates to the pilot that separation services are terminated. The SATS pilot acknowledges the transmission and descends into the SCA from the transition fix to the lowest available altitude. This availability will occur as the preceding aircraft also descends or begins the approach (figure 4). 


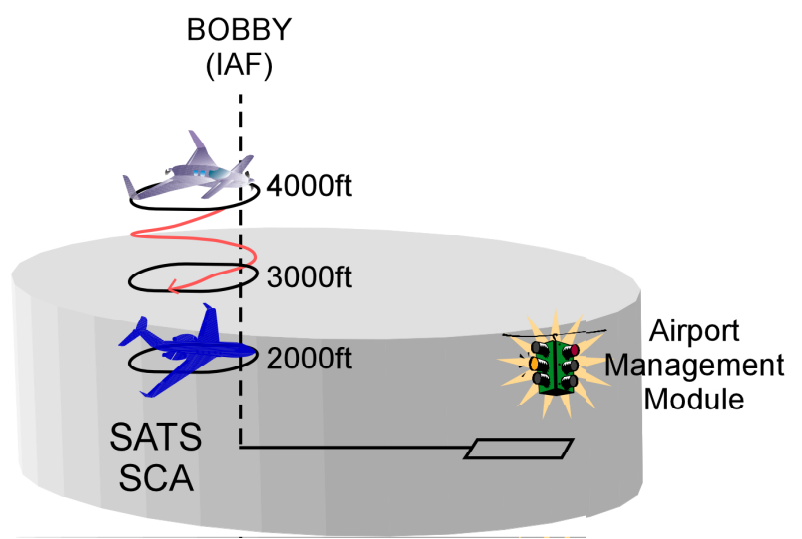

Figure 4. Entry into the SCA at the transition fix

As stated previously, pilots continue down the stack until they arrive at the initial approach altitude (2000 feet) at the IAF (BOBBY). Before leaving the hold and initiating the approach, the pilot must determine if the preceding aircraft is sufficiently ahead based on a selfspacing interval. All SATS aircraft will be able to selfspace using a baseline procedure. This baseline procedure would be to delay at the IAF and not begin the approach until spacing with the lead aircraft meets specified spacing criteria. SATS pilots desiring greater efficiency will be helped through onboard tools that enable the pilot to dynamically manage spacing. Selfspacing determination is based on the SATS aircraft's own planned performance, the actual and planned performance for the preceding aircraft, the approach geometry, wind conditions, and other factors. Once the spacing criterion has been met, the pilot leaves the hold and initiates the approach (figure 5).

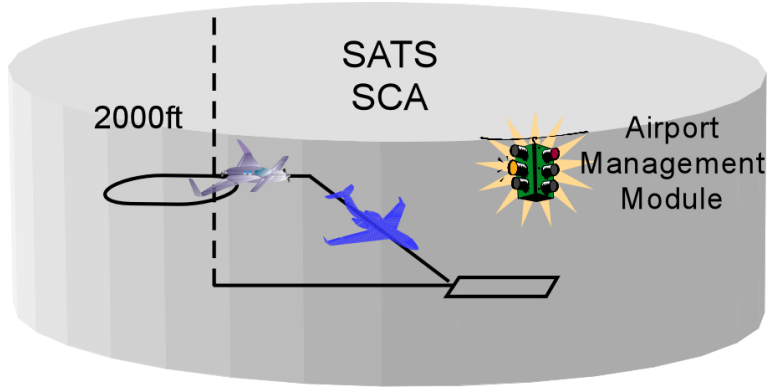

Figure 5. Initiating the approach

During the approach, the SATS aircraft continuously monitors the relative spacing between it and the preceding aircraft. If the following aircraft is predicted to get closer than the nominal spacing, then an alert is given to reduce its approach speed (figure 6).

Upon reaching the missed approach point, if the pilot has the runway in sight, the pilot performs a normal landing and exits the runway. If the aircraft cannot land, then the pilot follows the missed approach procedure, received upon entry to the SCA, to the missed approach holding fix (figure 7).

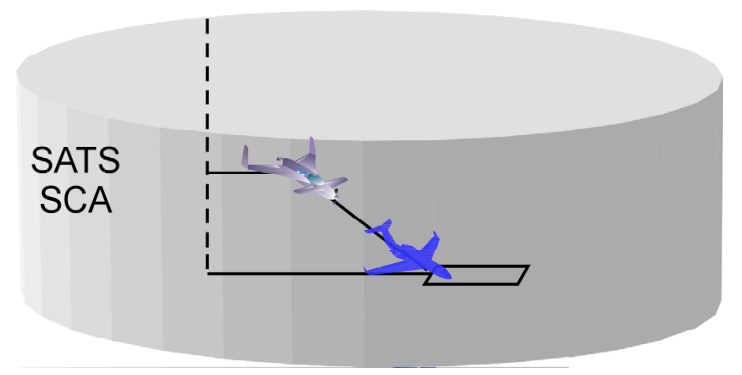

Figure 6. Flying the approach

Missed Approach - Prior to issuing a CTFN, the AMM must determine a conflict-free missed approach path for the SATS aircraft. A crucial element of this deconfliction is that the AMM will not assign two consecutive aircraft to the same missed approach path, ensuring that a performance disparity between the two aircraft does not cause conflicts. The SATS missed approach fixes will nominally be the two outrigger fixes of the GPS "T" on the departure side of the runway from the inbound approach path (ELLEN and GINNY in figure 1). Aircraft on a missed approach will go to the lowest available hold altitude, simplifying the transition back to begin another approach. If a missed approach is required, the SATS aircraft, as in normal IFR procedures, may begin a climb to the missed approach altitude at any point along the instrument approach path prior to the missed approach point (MAP).

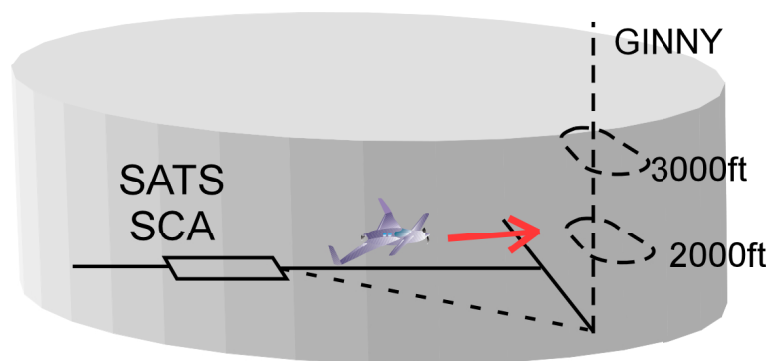

Figure 7. Flying the missed approach

Departures - A pilot who wants to depart the SATS designated airport files a normal IFR flight plan that includes a designated SCA departure fix with an associated departure hold (figure 1). Similar to today's operations at remote airports, approximately 30 minutes prior to the planned departure time, the pilot contacts ATC or flight service to obtain an expected clearance and follows this with a request for a departure from the AMM. This departure request includes the planned departure time and departure fix. 
The AMM responds with a CTFN message and the appropriate departure fix. As with arrivals, the CTFN is used to indicate relative sequence to the pilot. The airplane "to follow" might be on approach or an aircraft waiting to depart. If there is a delay required to accommodate arriving aircraft, the AMM will issue a delay time to the departing aircraft. It should be noted that the departure procedure is very similar to the arrival procedure.

Once the airplane the pilot is supposed "to follow" has either landed, performed a missed approach or departed, the pilot takes off and follows a SATS departure procedure to the departure fix, climbing to the highest available SCA holding altitude. As stated previously, to fly to the departure fix at 3000 feet, aircraft must meet an altitude crossing restriction on the climb out to the fix (eg., must be at or above 3000 feet 10 miles from the airport) While climbing out on the departure procedure, the pilot contacts ATC and requests release into ATC airspace from the departure fix. The pilot's expectation from ATC is to receive "released as cleared" to his destination. If ATC doesn't provide a release, ATC provides an expected release time and the pilot enters the hold at the departure fix, remaining within the SCA, until ATC can accept him. The ATC workload for receiving this aircraft into the IFR structure should not exceed that of a typical handoff from an adjacent sector, since the SATS aircraft will be on a filed IFR flight plan and transmitting an assigned transponder code.

In the event that the departing aircraft must immediately return to the airport, the pilot has the option to request a landing sequence from the AMM. The procedure and the supporting AMM operation will be very similar to a missed approach operation.

\section{Mixed-Equipage}

It is unrealistic to assume that by 2010 all aircraft wanting to operate at these airports will have the equipment required for performing SATS operations. While it will be possible for SATS aircraft to separate themselves from other SATS aircraft in the SCA, SATS aircraft can not separate themselves from non-ADS-B equipped aircraft that may want to operate within the SCA, since these aircraft will not be transmitting surveillance data. The only way non-SATS equipped airplanes could safely perform operations at this airport would be through today's system of procedural separation.

The SATS concept of operations will therefore enable two different sets of operations at an airport during IMC: SATS operations and today's procedural separation. However, these two different provisions for separation assurance cannot occur simultaneously. While one or more SATS aircraft are operating in the SCA during IMC, traditional IFR operations will not be permitted. When procedural separation is in effect, SATS operations will not be permitted and all other aircraft must be excluded from the SCA ("sterilization" of the airspace) on a one-in or one-out basis.

Whether the operation will be traditional or SATS will depend on the service requested by the pilot. This request is initiated when an aircraft approaches the SCA and the AMM is notified either by the SATS aircraft or ATC of the aircraft's intent to land. SATS equipped aircraft will notify the AMM directly and receive sequencing based on SATS capabilities during which other SATS operations are allowed but traditional IFR will be excluded. For traditional IFR, ATC will notify the AMM, which will incorporate the request into the departure/arrival queue. Traditional IFR operations commence when all SATS operations have been completed. When the SCA is cleared of SATS operations, the traditional IFR aircraft is permitted to enter the SCA to the exclusion of other aircraft (either traditional IFR or SATS). Upon conclusion of a traditional IFR operation, the SCA is cleared, opened, and service can then resume for another traditional IFR or SATS operation.

To facilitate mixed equipage operations, coordination between the AMM and ATC will be necessary. The ground link proposed in the AMM characteristics section of this paper could be used to fulfill this role. Using this link, ATC could suspend SATS operations to permit a traditional IFR operation. This suspension could only occur after all ongoing SATS operations have been completed. Failure of the link would then limit operations to SATS-only operations, assuming that the goal is to enable a greater number of operations at these airports using the SATS concept.

Non-Normal and Rare-Normal Operations

Non-normal and rare-normal conditions are characterized by a situation or configuration of the airplane or the environment that would, respectively, not normally or rarely be experienced during routine flight operations. Examples of non-normal operations would include systems failures such as communications, navigation, surveillance (ADS-B, TIS-B signals), and some aspects of automation such as system alerting failures to the pilot, the AMM and/or the controller. Rare normal operations would encompass extreme conditions such as wind shear and pilot errors, controller errors, and hazardous weather.

To mitigate these situations, procedures and technologies would be implemented to allow for a 
graceful degradation to a safe transition employing other than standard SATS procedures. Rules of the road to accommodate other than normal operations would be in place to assure safety during non-normal and rare normal operations. Separation breaches detected by systems technologies would be managed by individual aircraft depending on position and right of way rules that establish approach priority. Failures involving air service provider communications might include reversion to procedures similar to current day interventions by controllers. Hazardous weather (thunderstorms, icing, turbulence) on approach may require deviations or missed approaches. Weather graphics via data link might indicate the relative position of these conditions to the aircraft with the pilot executing deviations in accordance with approach procedures and maintaining separation.

\section{$\underline{\text { Simulation Capability }}$}

A software simulation capability is currently being developed to support the research and development of SATS concepts with the objective that simulation studies will support the design and analysis of approach and departure procedures in non-radar airspace at nontowered airports. Feasibility, performance, integration, and human factors issues will also be addressed as part of the SATS project. Both batch experiments and piloted simulation studies are being designed to answer the many open questions posed by the concept of operations.

The software platform currently implemented in the Air Traffic Operations Lab (ATOL) at NASA Langley, is an HLA (High Level Architecture) based system that includes an airport module modeling an automated sequencing facility and general aviation aircraft modules equipped with airborne conflict detection, alerting, and resolution logic.

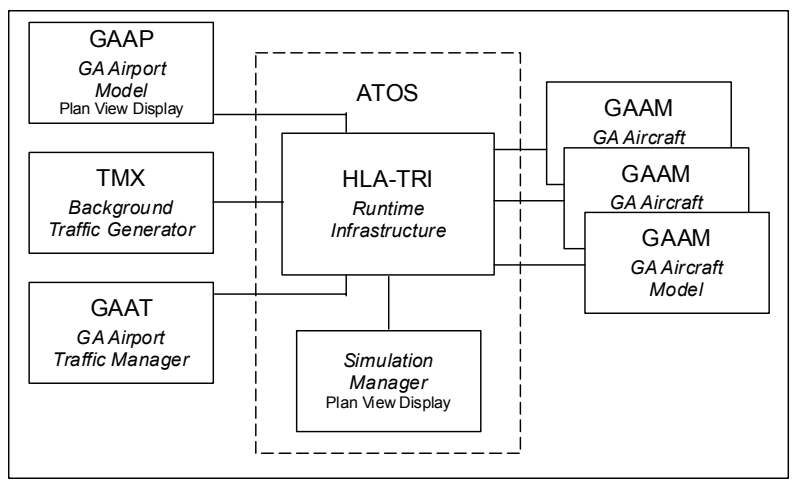

Figure 8. SATS Software Simulation Environment
Figure 8 is a diagram of the current implementation of the SATS simulation architecture. The Air Traffic Operations Simulation (ATOS) module provides connectivity to the distributed components using a High Level Architecture Runtime Infrastructure (HLA-RTI) that implements the system-wide communication and synchronization logic. A simulation manager permits the configuration and runtime monitoring of simulation experiments. The system includes a GA Airport Model (GAAP), a GA Airport Traffic Manager (GAAT), a background traffic generator (TMX), and multiple GA Aircraft Model (GAAM) or pilot stations.

The GA Airport Model (GAAP) is a software simulation of an automated air traffic sequencing facility that provides sequence advisories to arriving aircraft via a simulated data link. The GAAP main functions are to implement the communication protocol between participating aircraft and to determine their relative sequencing based on their current position and velocities. The GAAP design includes a flexible, generic approach definition that can be configured to reflect different experiment scenarios. The plan view display (figure 9) includes the approach procedure and all aircraft under ADS-B surveillance. The SCA is not shown but its boundaries can also be configured to model airports with different airspace and terrain constraints. Air traffic in the simulation includes background aircraft flying over and around the SCA and participating aircraft simulated by the GA Aircraft Models (GAAM).

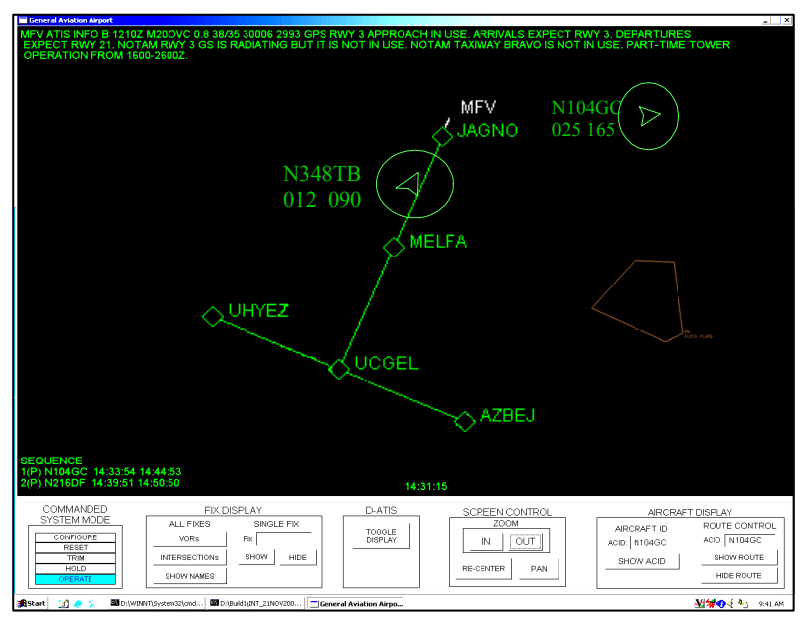

Figure 9. GAAP Plan View Display

The GAAM is a medium fidelity software simulation of a general aviation aircraft. Currently, the performance model and cockpit instrumentation are based on a Lancair Columbia 300 aircraft. Future revisions of this software will include additional aircraft models. A Navigation Management System (NMS) provides 


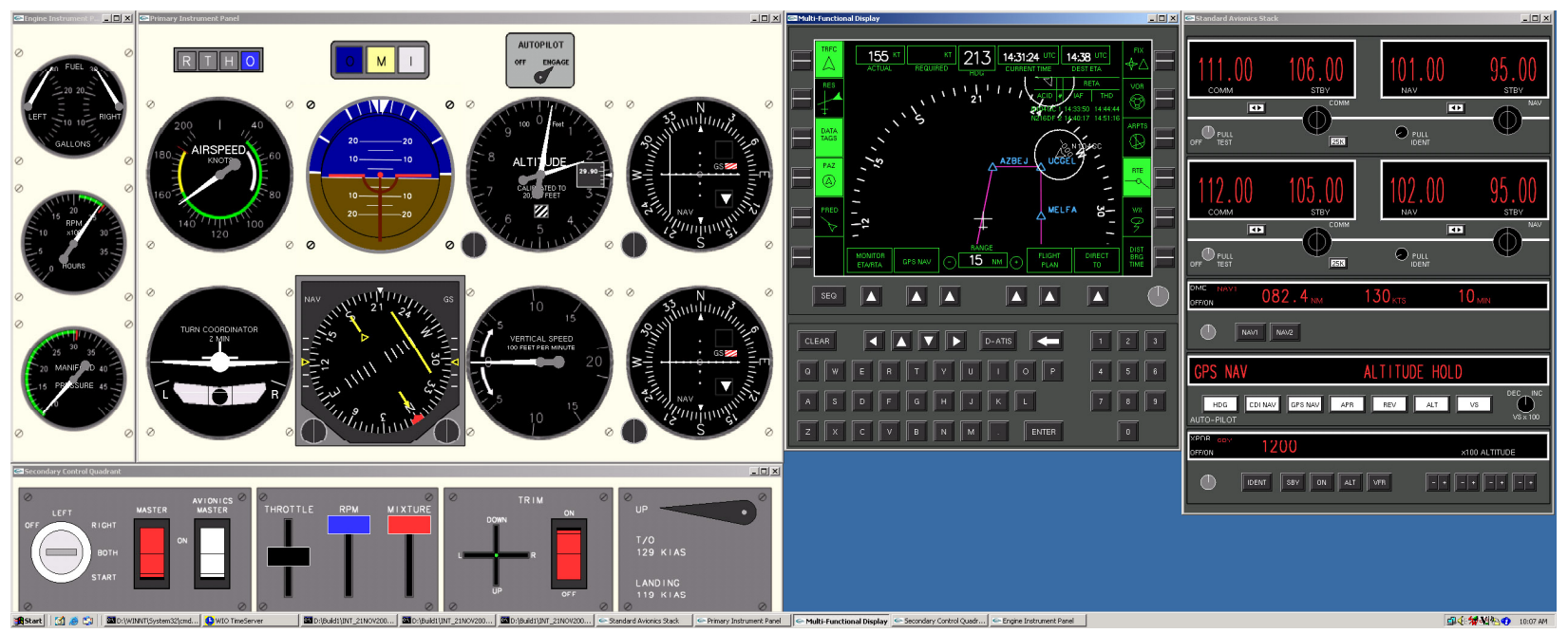

Figure 10. GAAM Instrument Panel Layout

navigation and lateral guidance functionality to allow the aircraft to follow a flight plan route. The NMS includes a flight planner that permits flight plan loading and editing. The GAAM instrument panel layout utilizes two monitors (figure 10). The left monitor contains the primary flight display, engine instrumentation, and flight controls. The right monitor contains a radio stack that includes GPS, audio panel, autopilot, and transponder and a multi-function display (MFD). The MFD primary screen shows a moving map display that includes navigation, CDA\&R, weather, and airport sequencing information.

\section{Summary}

The ability to operate multiple small aircraft, in near all weather conditions, at virtually any small airport, offers a unique opportunity for revolutionary transportation growth and passenger convenience. As part of this vision, this paper attempts to answer the question "can we do better than one at a time?" for aircraft operating under IFR at airports where currently operations are restricted to the inefficiency of a "one-in/one-out" procedure. The concept described in this paper is one solution to that problem. This concept will enable us to break the current day paradigm by applying emerging airborne and ground-based technologies to allow simultaneous operations by multiple aircraft in nonradar "terminal" airspace around small non-towered airports. The general philosophy underlying this concept is the establishment of a newly defined area of flight operations called a Self-Controlled Area (SCA). During periods of IMC, a block of airspace will be established around these SATS designated airports. Within the SCA, pilots, using advanced airborne systems, will have the ability and responsibility to maintain separation between themselves and other similarly equipped airplanes. Aircraft operating in this airspace will need special avionics to participate that will probably include ADS-B, communications data link, and appropriate self-separation tools. This concept also requires a new ground-based automation system typically located at the airport that will provide appropriate sequencing information to the pilots for safe and efficient operations.

This proposed concept emphasizes the integration with current and planned near-term NAS operations and systems. Additionally, the focus of the design approach is on simplicity from both a procedural and systems requirements standpoint. It is also assumed that any additional ATC workload must be minimized and that enroute procedures must be compatible with, and as similar to today's system as possible. This concept is based on a distributed decision-making environment that will provide pilots the necessary procedures, tools, and information to enable safe operations within the SCA while making minimum requirements of the ground support tool. While this is a distributed decision-making environment, the majority of the decision-making responsibility remains with the pilot.

The SCA described in this paper is intended to be a starting point for additional designs and analyses. No attempts were made to optimize the size or shape of the proposed airspace. To date, the development focus has been on providing an operational concept that was safe and effective, would enable more than one operation at a time, and would not require significant ground infrastructure costs or improvements. Additional research should be done to improve this initial design, both in terms of optimizing the SCA geometry and increasing capacity of the SCA. Additionally, batch and human-in-the-loop experiments must be performed to verify the concepts of operations. It is noteworthy, however, that this concept could be implemented and 
would enable more than one operation at a time. Operational concepts, such as the one proposed here, would enhance the opportunity for point-to-point air taxi or charter operations into smaller airports, providing greater convenience to the traveling public.

\section{Acknowledgements}

The authors wish to thank and recognize those who have contributed to the concept proposed and the production of this paper. Specifically we would like to thank Sally Johnson, John Koelling, Gary Lohr and Gary Millsaps for their insight and advice.

\section{$\underline{\text { References }}$}

1. Long, D., Lee, D., Johnson, J., and Kostiuk, P., “A Small Aircraft Transportation System (SATS) Demand Model." NASA/CR-2001-210874, June 2001 .

2. National Civil Aviation Review Commission, Mineta, N., Chair, "Avoiding Aviation Gridlock: A Consensus for Change." Sept 10, 1997.

3. RTCA Free Flight Steering Committee Report, "National Airspace System; Concept of Operation and Vision for the Future of Aviation." RTCA, Inc., Washington, DC, November 2002.

4. Federal Aviation Administration, "National Airspace System Operational Evolution Plan, Version 5.0; A Foundation for Capacity Enhancement 2003-2013." Washington, D.C., December 2002.

5. FAA AUA-600 Capstone ADS-B Evaluation Acceptability Memo, US DOT, December 2000, www.alaska.faa.gov/capstone/docs/lacey.pdf.

6. Ballin, M., Hoekstra, J., Wing, D., and Lohr, G., "NASA Langley and NLR Research of Distributed Air/Ground Traffic Management." AIAA Paper 2002-5826, October 2002.

7. Kuchar, J., and Yang, L., "Survey of Conflict Detection and Resolution Modeling Methods." AIAA Paper 97-3732, 1997.

8. Williams, D., "Time-Based Self-Spacing Techniques Using CDTI During Approach To Landing in a Terminal Area Vectoring Environment.” NASA-TM 84601, April 2000.
9. Cargo Airline Association and FAA Safe Flight 21 Office, "Phase 1 Operational Evaluation Final Report.” Wilmington, Ohio, April 2000.

10. Tobias, L. and Scoggins, J., "Time-Based Air Traffic Management Using Expert Systems." NASA TM-88234, Ames Research Center, Moffett Field, CA, April 1986.

11. Morgenstern, B. and Telsch, R. "A Description of the Phase I Automated Terminal Services Concept.” FAA-EM-76-6, November 1976.

12. Conway, S., and Consiglio, M., "A Method Of Separation Assurance For Instrument Flight Procedures At Non-RADAR Airports." AIAA2002-4448, August 2002.

13. Cain, J., "Multiple, Simultaneous Instrument Approaches and Departures in Instrument Meteorological Conditions at Airports Without Control Towers or Radar Service." Unpublished manuscript.

14. Federal Aviation Administration, "Terminal Arrival Area Design Criteria." 8260.45A, July 2000 .

15. Federal Aviation Administration, "Aeronautical Information Manual, section 5-4-9." United States Department of Transportation, 2003. 